\title{
FATS IN HUMAN NUTRITION
}

\section{A}

SYMPOSIUM on triglyceride fat in human nutrition was held at a meeting of the Nutrition Society in the Medical School, University of Birminghampen October 15. Sir Leonard Parsons, emeritu. professor of pædiatrics and dean of the Facul ty of Medicine, was in the chair.

The opening papef, on the chemical constitution of natural Mats, gas given by Prof. T. P. Hilditch (Live topl). He detailed the characteristic component fatty feids of animal depot fats, liver fats and milk fats. He directed attention to the preponderance in animal depot fats of oleic, stearic and palmitic acids, the latter forming in general 27-30 per cent of the total acids. Milk fats show similar constancy in palmitic acid content (slightly less than in depot fats) and in many mammals are closely similar to their depot fats in composition. In many other milk fats, especially those of ruminant animals, notable proportions of short-chain fatty actds are found. Phosphatides (animal or vegetable) contain palmitoleic acid and very long chain $\left(\mathrm{C}_{20}\right.$ and $\left.\mathrm{C}_{22}\right)$ unsaturated acids, and in these respects resemble the fatty acids found in fish oils and other fats of aquatic origin. In contrast, the component acids of vegetable fats cover a wide range and in general are far removed from those found in animal organs and depots. Prof. Hilditch emphasized the need for definition of specific fats in terms of their component acids rather than by average analytical characteristics such as iodine value or mean molecular weight, and indicated the extent to which this might be effected, when only small amounts of material are available, by the use of recent methods of separation of fatty acids. He showed how some of the characteristic features of certain depot and milk fats can possibly be explained on the basis of changes due to bio-hydrogenation or oxidation-reduction of already formed mixed palmitounsaturated (oleo)-glycerides.

Prof. P. Kraut (Dortmund) gave the second paper, on synthetic fats. Prof. Kraut described how the synthetic fatty acids are prepared from the wash liquors of the Fischer-Tropsch benzene synthesis. The mixture contains fatty acids with both odd and even numbers of carbon atoms. Nearly all the fatty acids are short-chain compounds up to $\mathrm{C}_{12}$. Small amounts of unsaturated fatty acids are eliminated by hydrogenation. It is also necessary to get rid of hydroxy fatty acids, the presence of which, even in small quantities, may affect the taste of the fat.

The synthetic glyceride esters of these fatty acids are readily hydrolysed by lipase-rather more rapidly than many natural fats because of the high proportion of short-chain fatty acids. Studies in animals and in human subjects have shown that they can satisfactorily replace natural fats without affecting the growth-rate or metabolic balance. The presence of branch-chain fatty acids may become troublesome, since these substances can give rise to tissue reactions ; and a method has been devised for the elimination of iso-acids from the fatty acid mixture. From the economic point of view, fat synthesis is at present expensive as compared with the cost of natural fats.

Dr. J. M. French (Birmingham) and Dr. H. G. Sammons (Birmingham) described methods which have been developed for the quantitative and qualitative investigation of fat absorption. The general quantitative assessment of fat absorption is most usefully investigated by daily estimation of total fæcal fatty acid in subjects on a measured fat intake. Further information can be gained from studying the effects of increasing the dietary fat or altering its chemical constitution. Methods for the detailed study of each phase of fat absorption (intraluminar, cellular and distributive) are based on experimental studies in animals and man. Intestinal intubation studies before and after feeding fat provide inform. ation as to the environmental conditions in the intestinal lumen and the preparation of ingested fat for absorption. In particular, the $p \mathrm{H}$, presence or absence of lipase, amylase, trypsin, phosphatase and lysozyme, and the degree of emulsification of the fat, have been studied by this method. The cellular phase is difficult to study in man, but some information may be obtained from the investigation of absorption of other substances. In the distributive phase, the occurrence of systemic post-absorptive hyperlipæmia can be determined by chylomicrography or serial micro-estimation of blood fat. Certain associated changes were also studied, especially gastric secretion and motility and intestinal mucous secretion. The former was investigated by standard methods and the latter by radiographic studies of the small intestinal pattern. From these various investigations it is possible to determine whether there is an overall defect in fat absorption, whether particulate (glyceride) absorption is normal, with a marked systemic post-absorptive hyperlipæmia, and whether nonparticulate (fatty acid) absorption is occurring to a greater extent, than normal, with resultant depression of gastric acidity and motility and increase of mucous secretion.

Prof. A. C. Frazer (Birmingham) spoke of the application of these various methods to the study of fat absorption in normal and abnormal human subjects. In the normal case 95 per cent or more of ingested fat is absorbed. Fat loading of the diet or the injection of a fat with a relatively high saturated. fatty acid content makes little difference to the amount absorbed. In normal circumstances, longchain triglycerides appear to be absorbed mainly in particulate form, and marked effects of free fatty acid absorption have not been observed. Hydrolysis of triglycerides containing short-chain fatty acids is more rapid and their liberation into the water phase more easily achieved. In normal subjects, the administration of fats containing a high proportion of short-chain fatty acids, such as milk fat, is likely to result in evidence of increasing fatty acid absorption, such as delayed emptying of the stomach, decreased gastric acidity, and radiographic changes of the intestinal pattern due to increased mucous secretion.

Cases of defective fat absorption, Prof. Frazer pointed out, can be broadly divided into two groups. In the first group, the overall fat absorption-defect is marked, less than 50 per cent being absorbed. Absorption is not proportional to intake, and intubation studies have shown faulty emulsification and hydrolysis. Such cases are associated with lack of bile salts or lack of pancreative lipase. Gastric acidity and emptying time, the radiographic intestinal pattern and glucose tolerance curves are normal. The evidence suggests that particulate absorption is prevented by faulty intraluminar emulsification, and fatty acid absorption in the upper intestine is prevented by delay in the hydrolysis and liberation of 
fatty acids, due to lack of lipase or bile salts. Hence there is a marked defect of absorption and no evidence of increased fatty acid absorption. The second group of fat absorption-defects usually shows a less marked decrease, 50-70 per cent being absorbed. Intraluminar emulsification is apparently normal. Consequently, hydrolysis and increased fatty acid absorption occur in the upper part of the intestine, giving rise to changes in gastric acidity and motility and increased mucous secretion. Decreased gastric acidity and motility and interference with absorption favour the upgrowth of intestinal flora; interference with carbohydrate absorption in particular provides an opportunity for fermentation. Heavy bacterial growth in the upper intestine may give rise to competition with the host for common essential nutrients. Thus, the sprue syndrome may arise from secondary changes which follow interference with the particulate absorption of fat and consequent increase of fatty acid absorption.

Dr. J. J. Elkes (Birmingham), reading a paper on the use of fat in intravenous alimentation, said that, theoretically, fat might be given either as soluble lipoprotein complexes comparable to the invisible fat of blood plasma or as finely dispersed triglyceride emulsions, similar to hyperlipæmic blood. Too little is known at present of the structure or functions of plasma lipoproteins to make it possible to use them. Finely divided oil-in-water emulsions, however, can be prepared relatively easily. Oil-inwater emulsions occurring in vivo consist essentially of triglycerides in a protein environment. Their stability is dependent upon the nature of the interfacial film. The composition of this film has been studied by observing changes in dispersion and electrophoretic mobility over a $p H$ range, or in the presence of enzymes, such as $C l$. welchii lecithinase. Characteristic flocculation patterns have been demonstrated. These are used for the comparison of artificial and natural emulsions.

Emulsions for intravenous alimentation must be proved satisfactory from the pharmaceutical, physicochemical, antigenic and metabolic aspects. A particle size of $0.5-1 \cdot 0 \mu$ diameter is desirable, and the emulsion must be stable on storage and autoclaving and resistant to oxidation by air. The emulsions must not be flocculated, or damaged, by solutions such as protein hydrolysate or glucose, with which they may be mixed, or by plasma protein. The emulsions must be non-pyrogenic and should produce no local or general tissue reactions. Finally, the metabolic availability of the fat particles must be demonstrated experimentally.

Seventy-four emulsions have been prepared, using plasma protein, formol-treated bovine serum, protein hydrolysate, and dextran in varying combinations with bovine cord phosphatide, brain phosphatide, soya bean phosphatide and cholesterol. Freeze-dried human plasma has been found to yield finely divided emulsions which strongly resemble the natural chylomicron emulsion in physico-chemical properties. Provided that low concentrations of stabilizer are used $(0.4$ per cent plasma protein per 10 per cent oil) the emulsion can be sterilized by heat. Preparations of dextran and bovine cord phosphatide yield satisfactory 30 per cent oil-in-water emulsions, which are tolerated well in acute experiments in doses of $1 \mathrm{ml}$. emulsion per $100 \mathrm{gm}$. body-weight. These emulsions disappear from the circulating blood within $60 \mathrm{~min}$. of intravenous injection, and there is no demonstrable fat embolism. Dr. Elkes stressed the usefulness of these emulsions as a means of studying fat transport and deposition. In these systems the core and the stabilizing film of the emulsion particles can be varied independently. The use of labelled material might be helpful in determining the movement of fat from one part of the body to another. The experiments of Chaikoff, Stare and Shafiroff, and their respective collaborators, augur well for the use of oil-in-water emulsions for parenteral nutrition in man.

A general discussion followed on the matters raised in these five papers. The opening papers will be published in the Proceedings of the Nutrition Society.

\section{WORK OF THE ROCKEFELLER FOUNDATION DURING 1948}

$T$

HE review of the work of the Rockefeller Foundation during $1948^{*}$ opens with a tribute to the opgstan bing reviews which appeared over the signgtur or Tr. Raymond B. Fosdick since 1936 ; but hif Luccssor as president, Mr. Chester I. Barnard, wdr hily maintains the standard set by his immediate predecessor. Mr. Barnard points out that the purpose of the Foundation is to serve the welfare of mankind without national, sectarian or racial discrimination, and that, in a quite informal and undesigned manner, the Foundation has become one of the cross-roads of the scientific, educational and scholarly world. In seventeen countries the Foundation maintains more or less permanent headquarters or research centres. Its officers travel extensively, being in constant touch with scholars, educators, men of science, statesmen, men of affairs and churchmen who seek their detailed judgment and informal advice.

This is the background for the belief that the more personal estimate contained in the president's 'review' should be continued, and Mr. Barnard then gives a brief restatement of the policies of the Foundation and programmes before surveying the outstanding features of the year. In general, the support of the Foundation has been directed to purposes for which it is otherwise difficult to secure funds, and the support has been of an initial or catalytic character with the idea that what has been demonstrated to be useful should then be carried on by other means. Current and palliative types of philanthropy are accordingly left to others, because the needs they encompass are more generally recognized. Further, the Foundation selected as its primary interest the promotion of knowledge and its effective application to human interests. Mr. Barnard instances the history of penicillin as one example of the way in which modest sums made available to scientific research helped to develop a therapeutic agent of great importance.

The parallel with the policy which has guided the trustees of the Nuffield Foundation in Great Britain will be remarked at once, and the whole of this part of the report of the Rockefeller Foundation could well be cited in support of Lord Beveridge's argument as to the place and need for voluntary action in the world to-day (see Nature, October 29, p. 721). $\mathrm{Mr}$. Barnard remarks, moreover, that the Foundation is for the most part a disbursing rather than an oper. ating agency, giving financial support but leaving the execution of the project to governments, universities and other recognized institutions; and he

* Rockefeller Foundation. A Review for 1948. By Chester I Barnard. Pp. 71. (New York: Rockefeller Foundation, 1949.) 